\title{
Boyd Amputation Using the Tension Band Technique
}

\author{
Mauricio LD Mongon ${ }^{1}$, Aurelio L Sposito ${ }^{2}$, George MN Nunes ${ }^{3}$, Bruno Livani ${ }^{4}$, William Belangero ${ }^{5}$
}

\begin{abstract}
Background: Amputation at the level of the hindfoot results in an equinus deformity from an imbalance of muscle-tendons acting across the ankle. Boyd's reconstruction for hindfoot amputations is a well-known technique that retains the calcaneus and fuses it with the distal tibia at the ankle mortise. It provides an excellent weight-bearing stump and in most cases does not require an artificial limb but its use has been restricted because of the difficulty in obtaining high union rates in the tibiocalcaneal fusion.

Materials and methods: Five patients (four unilateral and one bilateral) underwent leg amputations from January 2012 to November 2013 using the Boyd technique for reconstructing the stump and were stabilized with a tension band. The study sample consisted of adult patients who had traumatic reasons for the amputation. Patients under 18 years old with a hindfoot that was inadequate for Boyd reconstruction (i.e., insufficient soft tissue coverage or no posterior tibial artery pulse) were excluded. One (case \#2) had systemic comorbidities (e.g., hypertension, diabetes, chronic vascular insufficiency) as well as being a smoker. Three men and two women (mean age 39 years; range 21-61 years) were included. Three patients underwent amputation on the right side, one on the left side, and one bilaterally (case \#5). All patients presented with Gustillo and Anderson IIIC open fractures. The mean time from lesion to amputation was 2.25 weeks (range 1-4 weeks). The mean follow-up duration was 16 (range 12-24) months. The post-surgery examination included a clinical examination and radiography. A 6 minute walk test (6 MWT) was performed on week 32 after the amputation. This study was carried out with the approval of our institution's ethics committee. All patients provided a written informed consent form in accordance with the World Medical Association and the Declaration of Helsinki.

Results: All six stumps fused successfully. The 6 MWT results were comparable to those found in the literature for other lower limb amputees. Conclusion: The tension band technique used as part of the Boyd amputation to achieve tibiocalcaneal fusion was effective in all five trauma patients. Sound fusion was achieved in all cases with the 6 MWT scores comparable to that in the literature. This technique should be considered an osteosynthesis option for the Boyd procedure.

Level of evidence: Level IV, retrospective study.

Keywords: Amputation, Cerclage wiring, Operative surgical procedure, Reconstruction.

Strategies in Trauma and Limb Reconstruction (2019): 10.5005/jp-journals-10080-1433
\end{abstract}

\section{INTRODUCTION}

\section{Background}

A lower limb amputation yields excellent results when used for the correct indications. ${ }^{1}$ Despite modern reconstruction techniques and replantation, preserving a severely traumatized lower limb can produce poorer functional results than amputation and prosthetic use. ${ }^{2}$ Talus-tibial disarticulation (the Syme procedure) is used at the ankle because it is very effective and the surgeon's learning curve for this operation is short. Unfortunately, long-term results show a high incidence of posterior migration of the fat pad which may lead for a consequent stump revision. ${ }^{3}$ Boyd's reconstruction operation for hindfoot amputations ${ }^{4}$ is a well-known technique that retains the calcaneus and fuses it with the distal metaphyseal tibia in the ankle mortise. It provides an excellent weight-bearing stump with no need for an artificial limb but its use has been restricted because of the difficulty of securing a successful tibiocalcaneal fusion (high nonunion rates). ${ }^{5}$ The aim of the present study was to describe the use of the tension band technique ${ }^{6}$ to achieve fusion in the Boyd amputation in six adults and to compare the 6 minute walk test (6 MWT) with the literature.

\section{Rationale}

Boyd's reconstructive amputation on the hindfoot provides an excellent weight-bearing stump without a need for an artificial limb or prosthesis. This is important where the cost, particularly in third world countries, of an artificial limb is prohibitive for most individuals. The main reason for a limited indication for this procedure is the high nonunion rate for the tibiocalcaneal fusion.
${ }^{1,3-5}$ Department of Orthopedics and Traumatology, Unicamp, Campinas, São Paulo, Brazil

${ }^{2}$ Department of Ortopedia e Traumatologia, Universidade Estadual de Campinas, São Paulo, Brazil

Corresponding Author: Aurelio L Sposito, Department of Ortopedia e Traumatologia, Universidade Estadual de Campinas, São Paulo, Brazil, Phone: +55 19 98173-0419, e-mail: aurelioortopedia@gmail.com

How to cite this article: Mongon MLD, Sposito AL, Nunes GMN, et al. Boyd Amputation Using the Tension Band Technique. Strategies Trauma Limb Reconstr 2019;14(2):102-105.

Source of support: Nil

Conflict of interest: None

This may be from a lack of suitable internal fixation from traditional hardware. The most reliable and effective option is external fixation (e.g., the llizarov fixator) but the recovery period is usually uncomfortable for the patient. ${ }^{7}$

The functional results of the 6 MWT-initially described for use in respiratory diseases as an alternative to the 12 MWT_-can be used to compare with the results from the literature for lower limb amputees even if the patients in this sample (qualifying by being amputees) did not use a prosthesis. ${ }^{8-12}$

\section{Materials and Methods}

\section{Study Design and Setting}

Five patients underwent foot amputations (four unilateral and one bilateral) from January 2012 to November 2013 using the 
Boyd technique for reconstructing the stump. Stabilization of the calcaneum to the tibia was with a tension band. ${ }^{13}$ The preoperative characteristics of the patients are presented in Table 1. The study sample consisted of adult patients who had severe trauma as the indication for the amputation. Patients under 18 years old or those with an inadequate remnant hindfoot to allow the Boyd reconstruction (e.g., insufficient soft tissue coverage or no posterior tibial artery pulse) were excluded. ${ }^{6}$ One case (case \#2) had systemic comorbidities (e.g., hypertension, diabetes, chronic vascular insufficiency) as well as being a smoker. Three men and two women (mean age 39 years; range 21-61 years) were included. Three patients underwent amputation on the right side, one on the left side, and one bilaterally (case \#5).

All patients presented with open fractures that were of the Gustilo and Anderson IIIC grade. The mean time from injury to amputation was 2.25 weeks (range 1-4 weeks). The mean follow-up duration was 16 (range 12-24) months. The post-surgery examination included a clinical examination and radiography. The $6-\mathrm{MWT}^{5,7,14}$ was performed during week 32 after the amputation (Table 2).

This study was carried out with approval of our institution's ethics committee. All patients provided a written informed consent form in accordance with the World Medical Association and the Declaration of Helsinki.

\section{Surgical Technique}

Each patient was positioned supine on a radiolucent operating table. The use of a tourniquet was not necessary. The forefoot was amputated in the usual manner and the open wound was washed and prepared for the sterile Boyd reconstruction procedure (Fig. 1A). The talus was removed and both malleoli and residual cartilaginous surfaces of the distal tibia and calcaneus were resected with a pneumatic saw to expose the cancellous bone (Fig. 1B). The ankle was aligned in the plantigrade position $\left(0-5^{\circ}\right.$ valgus, $0^{\circ}$ flexion, $5-10^{\circ}$ external rotation) to obtain the largest surface contact area between the tibial pilon and calcaneus (as in the original description by Boyd). Bone apposition and the foot position were checked intraoperatively using fluoroscopy. Once good reduction was achieved, a 3-mm Kirschner wire was inserted longitudinally along the axis of the ankle through the heel fat pad (Fig. 1C).

A single $4.5-\mathrm{mm}$ cortical screw was inserted through the calcaneus in the coronal plane. A second $4.5-\mathrm{mm}$ cortical screw was about $5 \mathrm{~cm}$ from the distal tibial metaphyseal surface (Fig. 1D). These screws were $4 \mathrm{~mm}$ longer than the ideal measurement to ensure that $2 \mathrm{~mm}$ protruded on each side thereby permitting the use of two 1.2-mm cerclage wires to anchor the screws. The tension band technique was performed with vise grips to achieve compression at the site. Before complete tension, the Kirschner wire was removed, and final compression was obtained (Fig. 1E). One extra $4.5 \mathrm{~mm}$ screw was introduced vertically between the calcaneus and tibia for additional compression and stabilization (Figs 1D and E). The surgical site was checked for haemostasis before wound closure absorbable stitches (Poliglactine 910 \#4.0) to avoid a hematoma. No significant blood loss occurred. The wound was closed without drains and a compressive cast dressing was applied below the knee for 3 weeks. Full weight bearing was encouraged with a walking cast after 3 weeks.

\section{Aftercare}

Oral opioid analgesics were prescribed every 12 hours if the patient had pain. Full weight-bearing was encouraged progressively and adjusted to the individual's pain and strength when walking with a walking frame or crutches. The walking cast was changed monthly and an X-ray taken until fusion was confirmed. Unrestricted weight bearing was permitted (without adaptations or walking aids) when the fusion was clinically and radiographically confirmed.

The mean follow-up duration was 16 (range 12-24) months. The post-surgery assessment included clinical examination and radiography. The $6-\mathrm{MWT}^{5,7,14}$ was performed at week 32 after the amputation and at 1 year. The variables recorded at assessment included state of fusion, results of the 6-MWT, and patient satisfaction with the procedure.

\section{Results}

A clinically and radiologically successful tibiocalcaneal fusion was achieved in all patients at a mean of 16.6 (range 13-28) weeks.

Table 1: Preoperative patient data

\begin{tabular}{llllll}
\hline Patient & Gender & $\begin{array}{l}\text { Age at amputation } \\
\text { (years) }\end{array}$ & Etiology & Side & $\begin{array}{l}\text { Time from lesion } \\
\text { (weeks) }\end{array}$ \\
\hline 1 & M & 23 & Traumatic & Left & 2 \\
2 & M & 52 & Traumatic & Right & 1 \\
3 & F & 38 & Traumatic & Right & 1 \\
4 & M & 61 & Traumatic & Right & 2 \\
5 & F & 21 & Traumatic & Bilateral & 4 \\
\hline
\end{tabular}

Table 2: Postoperative patient data

\begin{tabular}{llllll}
\hline Patient & Time to fusion (weeks) & $\begin{array}{l}\text { Follow-up time } \\
\text { (months) }\end{array}$ & Complications & Infection & 6MWT (metres) \\
\hline 1 & 14 & 24 & No & No & No \\
2 & 14 & 18 & No & No & 260 \\
3 & 12 & 16 & No & No & 256 \\
4 & 16 & 14 & No & No & 250 \\
5 left & 14 & 12 & No & No & 252 \\
5 right & 16 & 12 & & 252 \\
\hline
\end{tabular}



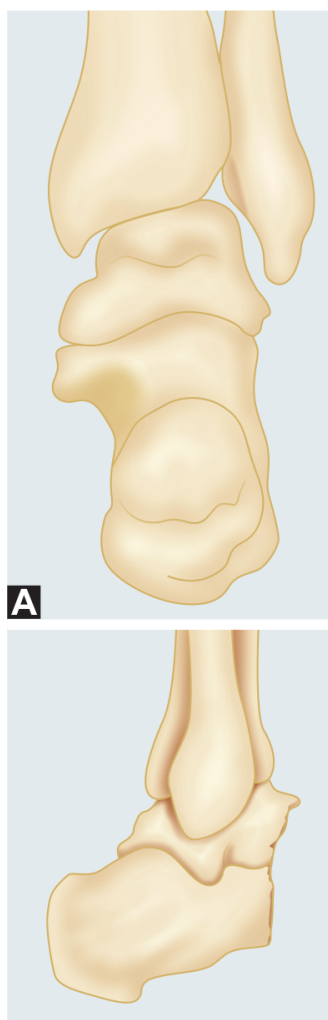
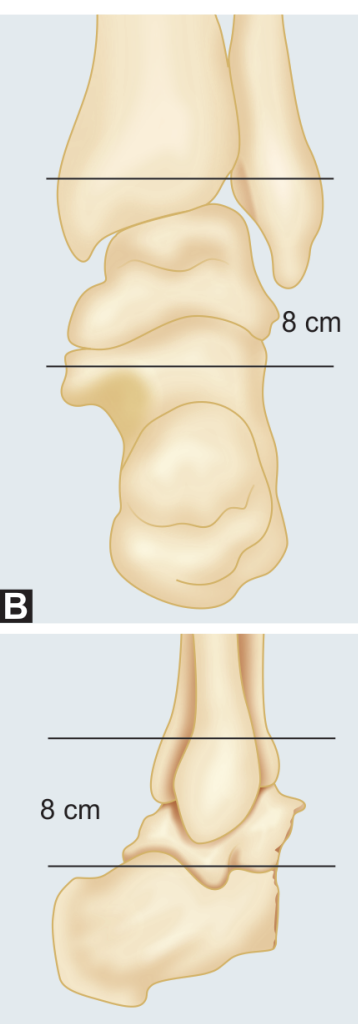
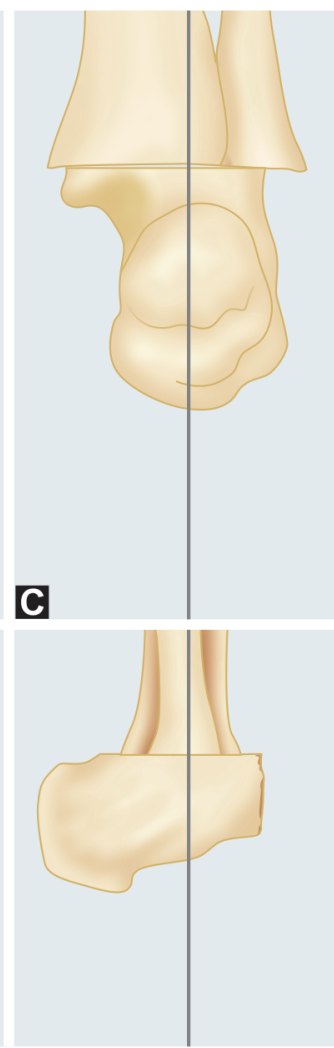
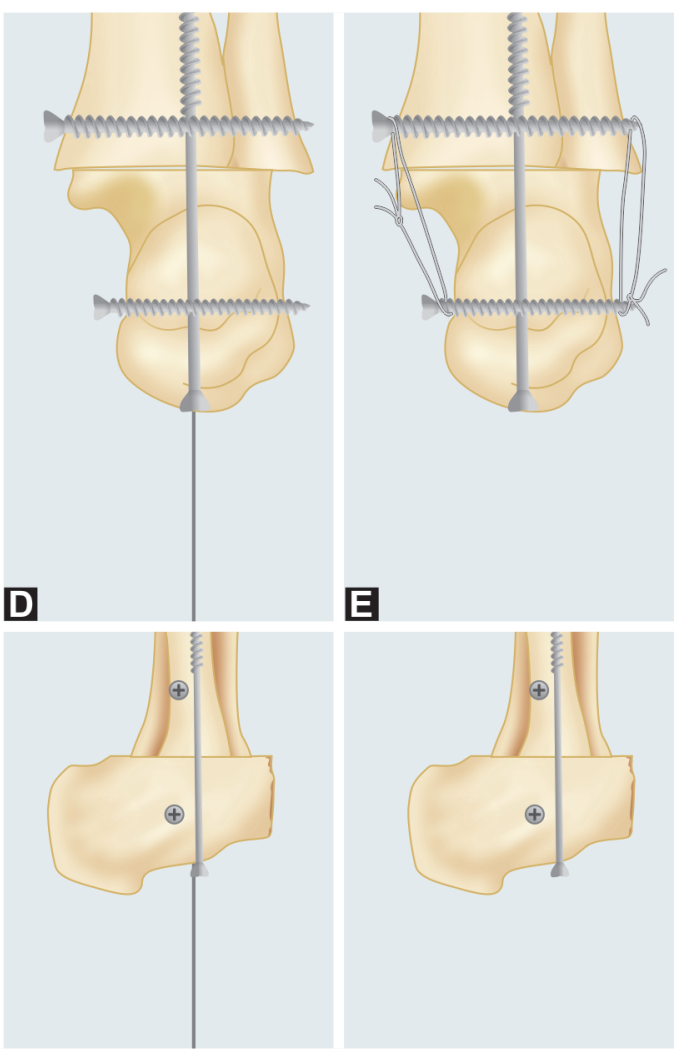

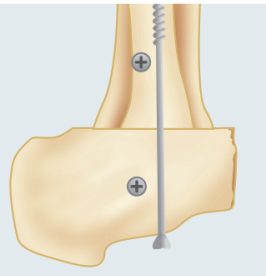

Figs $1 \mathrm{~A}$ to $\mathrm{E}$ : (A) Amputation of the forefoot, leaving the hindfoot intact. Anatomical representation; (B) Removing the talus and distal tibia and fibula to expose cancellous bone; (C) Ankle aligned in the plantigrade position ( $0-5^{\circ}$ valgus, 0 flexion, $5-10$ external rotation) and insertion of a $3 \mathrm{~mm}$ Kirschner wire; (D) Three $4.5 \mathrm{~mm}$ screws inserted (two cortical and one cancellous). The Kirshner wire remaining; (E) Compression and stabilization using a $1.2 \mathrm{~mm}$ cerclage wire
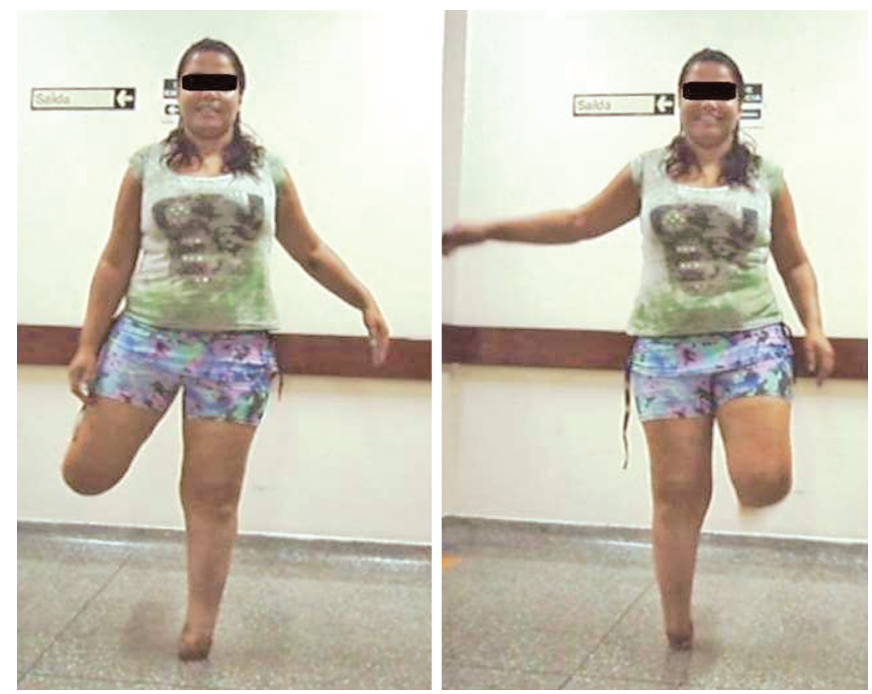

Fig. 2: Patient in full weight bearing without cast or crutches

Fusion was considered complete when patients were completely pain-free (Fig. 2). Additionally, no motion between the fragments could be clinically demonstrated and the radiographic examination showed the presence of trabecular continuity through the distal tibia and calcaneum.

The functional results as measured by the 6 MWT-initially described for use in respiratory diseases as an alternative to the $12 \mathrm{MWT}^{8,10-12}$ — consists of asking the patient to ambulate at normal speed for the maximum distance possible in a predetermined course, usually a corridor. ${ }^{15}$ When compared to the available literature on lower limb amputees (at different levels of amputation) or to transtibial amputees, we found that not only could the patients achieve scores comparable to those studies (that matched their daily living necessities) but they could do it without a prosthesis, walking an average of 310 meters (range 290-345 metres). ${ }^{16-18}$

\section{Discussion}

Good decision making is critical when managing severely traumatized lower extremities. Initial limb salvage is the preferred option for most patients. However, objective reasoning and judgment suggest that some cases are better served by limbablation procedures. Amputation must be considered a viable management strategy rather than a failure of treatment. ${ }^{19}$ The main objective of this work was to show a method to increase union rates in Boyd's technique, providing a reproducible, technically simple and useful tool for the management of a limb that was not suitable for salvage.

The major limitation of this study is that the total number of cases was very small (five patients, six stumps). The reason for this limited case series is that the inclusion and exclusion criteria limited the availability of cases as most cases with a mangled foot are often in need of a transtibial amputation. Another reason is that some patients did not agree to participate in the study, choosing 
the traditional Ilizarov or crossed $4.5 \mathrm{~mm}$ screw methods as their choice of fixation.

Boyd's reconstruction operation for hindfoot amputation was chosen because it provides an excellent weight-bearing stump with no need for an artificial limb, but its use has been restricted because of the difficulty achieving the tibiocalcaneal fusion (high nonunion rates). We have found that this is a promising fixation technique based on the effectiveness of dynamic compression at the desired fusion site.

The study has also shown that patients could perform the 6 MWT without need for prosthetics and with results comparable to that in the literature. Additionally we have preserved a sensate stump and with a high satisfaction level amongst patients.

The current study suggests that an inexpensive fixation device can be used successfully for tibiocalcaneal fusion in a Boyd amputation. It has been demonstrated to be a safe and effective surgical treatment option for post-traumatic mangled feet needing an amputation. It is also a simple, less expensive surgical procedure that allows early full weight-bearing and independent community ambulation after successful fusion.

\section{Compliance with Ethical Standards}

All procedures followed were in accordance with the ethical standards of the responsible committee on human experimentation (institutional and national) and with the Helsinki declaration of 1975 (in its most recently amended version). Informed consent was obtained from all patients included in the study.

\section{References}

1. Harris RI. Syme's amputation; the technical details essential for success. J Bone Joint Surg Br 1956;38-B(3):614-632.

2. Kornah B. Modified boyd amputation. J Bone Jt Surg 1996;78(1): 149-150. DOI: 10.1302/0301-620X.78B1.0780149.

3. Blum E. Boyd amputations In children. Clin Orthop Relat Res 1939. 138-143.

4. Kirkup J. Elective amputation: early evolution to the end of the 17th century. A history of limb amputation. London: Springer; 2007. 55-67.

5. Livani B, de Castro GF, Tonelli Filho JR, et al. Pedicled sensate composite calcaneal flap to achieve full weight-bearing surface in midshaft leg amputations: case report. J Reconstr Microsurg 2011;27(1):63-66. DOI: 10.1055/s-0030-1267831.
6. Mongon MLD, Garcia Costa KV, Bittar CK, et al. Tibiotalar arthrodesis in posttraumatic arthritis using the tension band technique. Foot Ankle Int 2013;34(6):851-855. DOI: 10.1177/1071100713483097.

7. Mongon MLD, Ribera FC, de Souza AMA, et al. Pedicled sensate composite calcaneal flap in children with congenital tibial pseudoarthrosis. J Pediatr Orthop 2017;37(4):e271-e276. DOI: 10.1097/ BPO.0000000000000942.

8. Butland R, Pang J, Gross ER, et al. Two-, six-, and 12-minute walking tests in respiratory disease. Britsh Med J 1982;284(6329):1607-1608. DOI: 10.1136/bmj.284.6329.1607.

9. Lin-Chan S, Nielsen D, Shurr D, et al. Physiological responses to multiple speed treadmill walking for syme vs. transtibial amputation-a case report. Disabil Rehabil 2003;25(23):1333-1338. DOI: 10.1080/09638280310001608618.

10. McGavin CR, Gupta SP, McHardy GJR. Twelve-minute walking test for assessing disability in chronic bronchitis. Br Med J 1976;1(6013):822823. DOI: $10.1136 / \mathrm{bmj} .1 .6013 .822$.

11. Montgomery PS, Gardner AW. The clinical utility of a six-minute walk test in peripheral arterial occlusive disease patients. J Am Geriatr Soc 1998;46(6):706-711. DOI: 10.1111/j.1532-5415.1998.tb03804.x.

12. Mungall IPF, Hainsworth R. Assessment of respiratory function in patients with chronic obstructive airways disease. Thorax 1979;34(2):254-258. DOI: 10.1136/thx.34.2.254

13. Nather A, Wong KL. Distal amputations for the diabetic foot. Diabet Foot Ankle 2013;4(1):4-7. DOI: 10.3402/dfa.v4i0.21288.

14. Mongon ML, Piva FA, Mistro Neto S, et al. Cortical tibial osteoperiosteal flap technique to achieve bony bridge in transtibial amputation: experience in nine adult patients. Strateg Trauma Limb Reconstr 2013;8(1):37-42. DOI: 10.1007/s11751-013-0152-0.

15. Anon. ATS Statement. Am J Respir Crit Care Med 2002;166(1):111-117. DOI: 10.1164/ajrccm.166.1.at1102.

16. Gailey RS, Roach KE, Applegate EB, et al. The amputee mobility predictor: an instrument to assess determinants of the lower-limb amputee's ability to ambulate. Arch Phys Med Rehabil 2002;83(5):613627. DOI: 10.1053/apmr.2002.32309.

17. Linberg AA, Roach KE, Campbell SM, et al. Comparison of 6-minute walk test performance between male active duty soldiers and servicemembers with and without traumatic lower-limb loss. J Rehabil Res Dev 2013;50(7):931-940. DOI: 10.1682/JRRD.2012.05.0098.

18. Wurdeman SR, Schmid KK, Myers SA, et al. Step activity and 6-minute walk test outcomes when Wearing low-activity or high-activity prosthetic feet. Am J Phys Med Rehabil 2017;96(5):294-300. DOI: 10.1097/PHM.0000000000000607.

19. Tarkin IS, Sop A, Pape H. High-energy foot and ankle trauma: principles for formulating an individualized care plan. Foot Ankle Clin NA 2008;13(4):705-723. DOI: 10.1016/j.fcl.2008.08.002. 